\title{
A Novel Approach of Attendance Display Classrooms Using Image Processing Technique
}

\author{
Anurag Sinha ${ }^{1}$, Arinjay Biswas ${ }^{2}$ \\ ${ }^{1,2}$ Department of computer science and IT, Student, Amity University Jharkhand \\ Ranchi, Jharkhand, India \\ Email: anuragsinha257@gmail.com¹, biswas87280@gmail.com²
}

\begin{abstract}
Participation in every film is free. The traditional approach is to register for the service to be attended to keep at the individual student. The biometric system is another measure of attendance. The implementation of biometric recognition systems to the physical or behavioural characteristics, such as iris, voice, face and fingerprints. However, these measures have proved-time consuming and boring. This makes significant progress in the stands automatically, marking the counting process through. The proposed method is aimed at the students present at the time, and it can be used by other teachers for verification. In this paper along with the CCTV cameras to capture entering students in the classroom and went out. Attendance is displayed by the numbers an hour from entering the class areas.
\end{abstract}

Keywords- Automatic attendance, Display board, CCTV, Counting

\section{INTRODUCTION}

Chat in a long procession of the flow process begins with recognizing what art can detect input from front to pilot diameter. In today's world, they are good for the students to engage in the kind shown when it is only effective in the classroom. The look of detection and our society is nothing. The human ability to recognize the human mind is the most remarkable. And it is surprising how the human mind can persist even among those along with the human person and others by passages, and depositing the form of slight changes. That the human mind is an excellent opportunity to generate about the same due to the positive image of the human face recognition, that is attracted significant attention from researchers to replicate the investment in time to researching and effective algorithms for facial recognition to electronic systems. for human use.

The process is said to find the face of face detection. Face to face with the investigation into the detection of different expressions, and he is the desirable corner background image in different sizes reports, the face of the parameters. The look identifies either one of those things that are made by analyzing the patterns in an image. Here, algorithms that are known to the process make use of the extract, as well as a database that matches.

This project has been highlighted by the use of Selections are ordinarily made it known that it is such, that the service of the readings. The use of clickers, swiping ID cards and manually jotting down the names on the paper so that the student to track and prompted them to ask to run the project. It appeared as if God's knowledge of a birth control method to detect the presence of the very being founded with the theme. The rest are as follows: Book Covers related work.

\section{RELATED WORK}

Different methods are used before students are present in class. A traditional method is to manually record the teacher's attendance. But the limitations of this approach may be the loss of attendance records in the future.

Another approach can be fingerprint-based attendance systems where a handheld device can Students are given fingerprints to indicate their presence. The advantage of this approach is that participation is done without the involvement of the instructor, and this technique guarantees signs of presence throughout the event. However, the problem with this approach is that it is difficult to scan the fingerprints of young people, and over time there can be changes in their fingerprints, and many people perceive them incorrectly. It is not suitable for the fingerprint recognition system

Attendance management systems based on radio frequency identification are also used. In this system, each student will have a unique card and the card will be exchanged for viewing on the machine. The switching unit is directly connected to the system where the current status data is stored. The limitation of the above approach is that even unauthorized people can participate. The iris-based presence system offers a solution to the above limitations as the eye is the most protected part of the body and cannot last a lifetime. However, facial recognition can be performed automatically using the camera module. This technique requires less storage space as it does not require the active participation of students to gain presence with the camera while capturing faces that will be stored on smaller devices.

\section{METHODOLOGY}

The classroom presence display system helps to detect the presence of students when using a Pi camera to recognize a face. There are several limitations to the existing system, so we propose this technique in a way that removes the limitations of the existing system. 


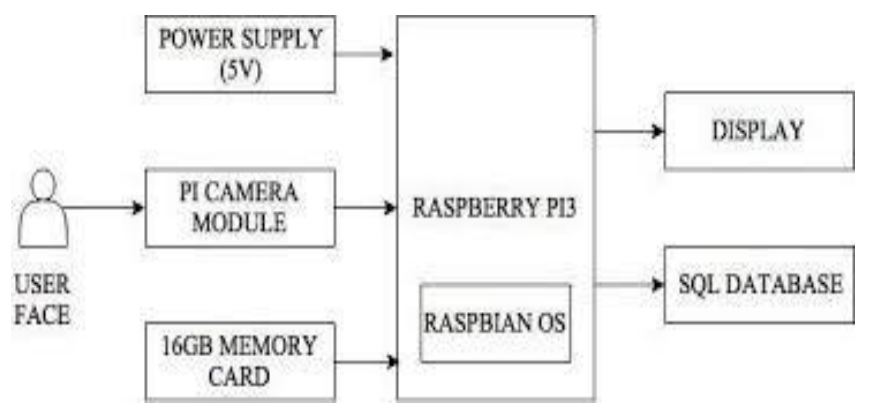

Fig 1: Diagram of Proposed System

The overall system is divided into three categories: database creation, dataset training, and testing.

1. Creation of the database

a) Camera initialization

b) Convert the image to greyscale and detect faces.

c) Save the given entry in the database using up to 20 frames as labels

2. Training

a) Initialize the LBPH face recognition device.

b) Import faces from the database folder to train LBPH face recognition software.

c) Save the trained data in XML or YAML file format.

3. Test

Load Haar classifier, LBPH face recognition to test the trained data

a) Image capture from the camera

b) Convert to greyscale.

c) Detect faces

d) Identify the face using the recognition device above. The system uses the Viola-Jones algorithm for face detection, which uses the Haar function for detection. The Raspberry Pi is the main component of this project. Capture images using a webcam or multi-camera module. To access the Raspberry Pi console, use a laptop or keyboard and mouse on a display device such as a TV connected to the pi.

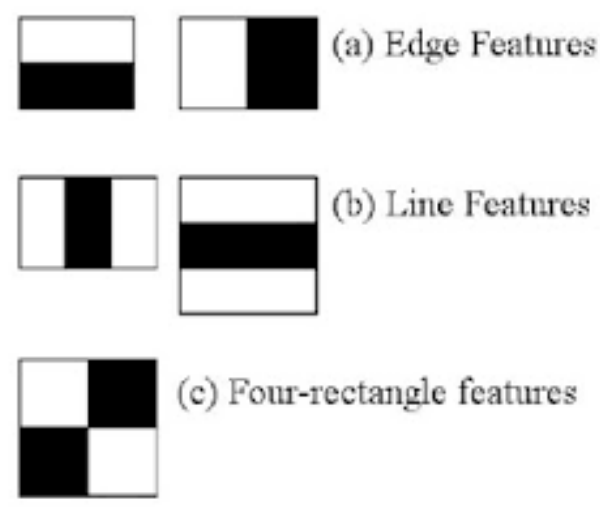

Fig 2: Sorts of Haar Features

Vol. 4 (4), October 2020, www.ijirase.com
Each person will create a value which is the difference between the sum obtained from the white pixels and the angle behind the sum of the rectangles. You can collect lots of features using all sizes and different positions of the sorter. It seems to have become one because the arithmetic number has been increased and the classifier calculation has been further improved. We apply this concept to move away from the whole picture. It is the provision of rectangular grids and algorithm arrangements that quickly and efficiently generate arrays of the highest values, filled with ideas from those people in a given space. And later in the image (x,y), this is the sum of the elements to the left of $(x, y)$.

$$
\begin{aligned}
& \text { ii }(x, y)=\Sigma \text { i }\left(x^{\prime}, y^{\prime}\right) \\
& x^{\prime} \leq x, y^{\prime} \leq y
\end{aligned}
$$

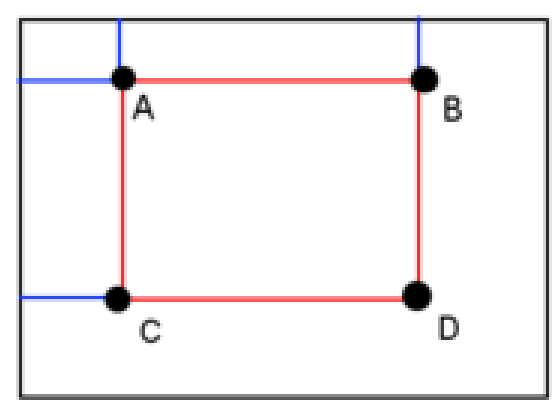

Sum $=\mathrm{D}-\mathrm{B}-\mathrm{C}+\mathrm{A}$

Fig 3: Integral Image

To understand more about the complexity of the classifiers are applied not only that a machine learning algorithm is to use a boost to Adam's disobedience, who is inbuilt in the Open cv Profile to the library of the classifiers, which classifier to eliminate due to the overflow. It features a classifier week probably fifty or through a process of deduction. A total of seven classifiers gives everyone a strong classifier which makes the process of deduction plan. In particular, the genus can not exist except in the choice men of the earth are not the first, that if we remove. We are not going to use it to his chosen country of the region, and how he, is discarded down according to the places, which bypasses through all the ie classifiers. All the strong classifiers are treated as a face uncovered. The face of it was not upon the face of the hand out of the recognition which results from the classifiers they are gone over time. At the time of this algorithm, we use binary Local Patterns (LBPH) for face recognition. Lbh simple as soon as the agents assigned by their resemblance to the image of adjacent pixels. Local integral picture of this model histogram found that underlies the binary representation of the decimal forms a complete image. Face recognition is the most susceptible to competition and changes the brightness of the face. The creator of the image of the face of a lot of them again, the outcome is uncertain, they acknowledge the fact that the face of the pre-processing is less of a luminous, you will see the image of the object of encouraging a constant 
position in the within the business, however, be any more. In this project, we use a histogram pre-processing face. We use the right hand to the pre-processing is separated, the efficiency of the face of my hand's stabilization.

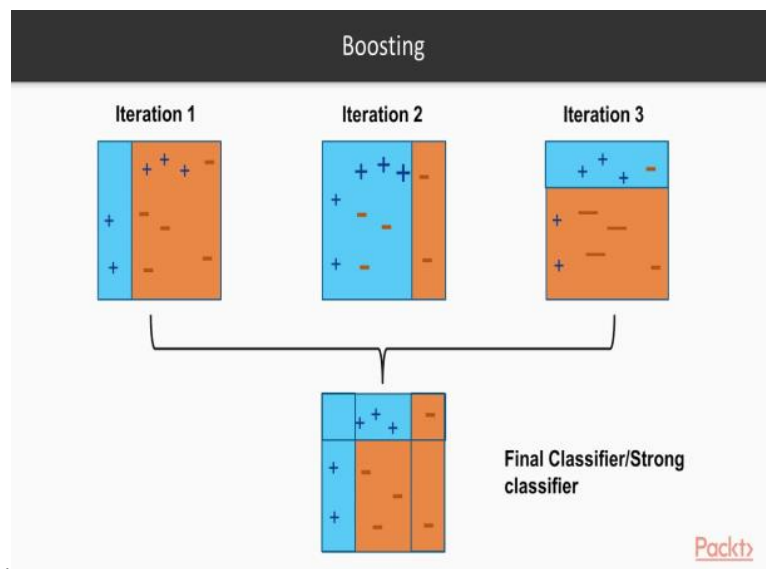

Fig 4: AdaBoost Classifiers

\section{EXPERIMENTAL RESULTS}

A series of experiments carried out on the data obtained from the project. System performance evaluation is done using datasets.

Below are screenshots of the various stages of the classroom attendance display system.

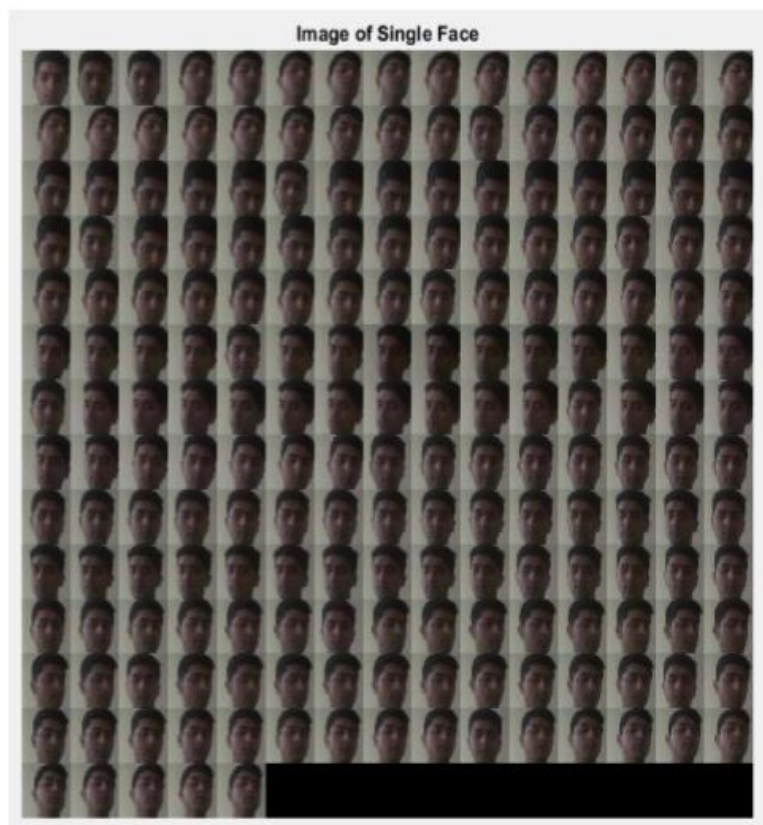

Fig 5: Dataset of a single person

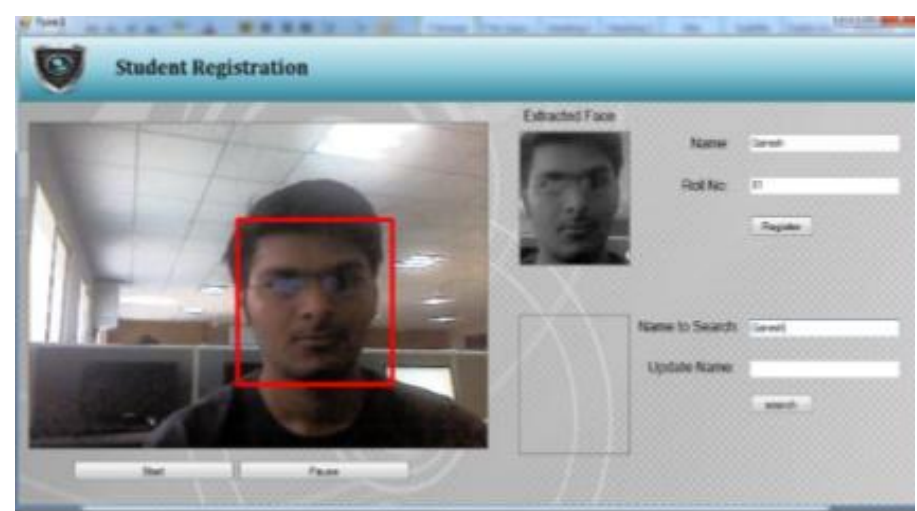

Figure 6 is the recording process performed in front of the Pi camera. At the time of the enrolment process, the phases of the student with different orientation are recorded

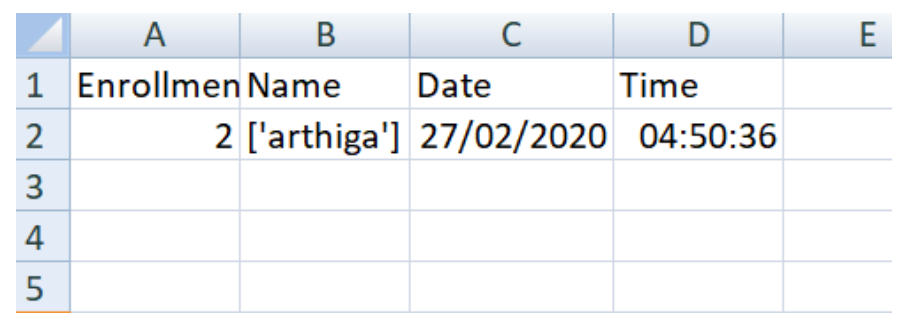

Fig 7: An automatic excel attendance sheet is generated after marking the attendance.

\section{CONCLUSIONS}

I understand that there is a wide range of methods, e.g. boring and counterproductive biometrics, RFID based attendance systems. To overcome the limitations of the dominant system, the above framework is proposed. This is the best and most robust arrangement for every time and every special security effort. Therefore, we built a secure engagement framework that implements an image processing algorithm that identifies faces in the classroom and accurately confirms attendance.

\section{FUTURE SCOPE}

The same design is often used for multiple security applications that require authentication to access each system's permissions. Facial recognition algorithms are often improved in terms of resource utilization so that designs can recognize multiple faces at once, making the system much better. These projects are often developed and used for home security, personal and organizational benefits. We will also quickly follow a specific student in the company with the help of this technology.

\section{REFERENCES}

[1] Lukas, S., Mitra, A. R., Desanti, R. I., \& Krisnadi, D. (2016, October). Student attendance system in classroom using face recognition technique. In 2016 International 
Conference on Information and Communication Technology Convergence (ICTC) (pp. 1032-1035). IEEE.

[2] Patil, M. R. V., \& Bangar, M. S. Video Surveillance Based Attendance system.

[3] Sharma, S., Gupta, T., \& Kumar, R. (2018). Face Recognition in Real Time for Attendance Marking System. International Journal of Scientific Research in Science and Technology.

[4] Veer, N. D., \& Momin, B. F. (2016, May). An automated attendance system using video surveillance camera. In 2016 IEEE International Conference on Recent Trends in Electronics, Information \& Communication Technology (RTEICT) (pp. 1731-1735). IEEE.

[5] Dhavalikar, A. S., \& Kulkarni, R. K. (2014, February). Face detection and facial expression recognition system. In 2014 International Conference on Electronics and Communication Systems (ICECS) (pp. 1-7). IEEE.

[6] Chatrath, J., Gupta, P., Ahuja, P., Goel, A., \& Arora, S. M. (2014, February). Real time human face detection and tracking. In 2014 international conference on signal processing and integrated networks (SPIN) (pp. 705-710). IEEE.

[7] Shirodkar, M., Sinha, V., Jain, U., \& Nemade, B. (2015). Automated attendance management system using face recognition. International Journal of Computer Applications, 975, 8887. 